\title{
Aminaftone in the Treatment of Raynaud's Phenomenon in Systemic Sclerosis: New Perspectives
}

\author{
Simone Parisi, Marco Scarati, Marta Priora, Clara Lisa Peroni, Angela Laganà, Enrico Fusaro \\ Department of General and Specialist Medicine, Division of Rheumatology, University Hospital Città della Salute e della Scienza di Torino, \\ Turin, Italy
}

\author{
Email address: \\ simone.parisi@hotmail.it (S. Parisi)
}

\section{To cite this article:}

Simone Parisi, Marco Scarati, Marta Priora, Clara Lisa Peroni, Angela Laganà, Enrico Fusaro. Aminaftone in the Treatment of Raynaud's Phenomenon in Systemic Sclerosis: New Perspectives. American Journal of Internal Medicine. Vol. 3, No. 5, 2015, pp. $204-209$.

doi: 10.11648/j.ajim.20150305.12

\begin{abstract}
Objective: The aim of this study was to assess the efficacy (in terms of improved VAS Pain, Raynaud's Phenomenon attacks frequency and Raynaud's Condition Score) of the Aminaftone in patients with Raynaud's Phenomenon (RP) secondary to Systemic Sclerosis (SSc). Methods: Open label controlled study. We evaluated the efficacy of Aminaftone in the treatment of secondary RP in 108 patients with SSc consecutively recruited and divided in two groups. Group A: 51 Patients in treatment with Calcium Channel Blockers, prostanoids (iloprost 2ng [ $\mathrm{min} / \mathrm{kg}$ every 4 weeks), Endothelin Receptor Antagonist and Phosphodiesterase-5 inhibitors (Standard of Care). Group B: 57 Patients in treatment with Calcium Channel Blockers, Prostanoids, Endothelin Receptor Antagonist and Phosphodiesterase-5 inhibitors and Aminaftone (Standard of care + Aminaftone). Results: The number of RP attacks in group treated with Aminaftone (Group B) was lower than the group treated with standard therapy (Group A) from baseline to Week 48, obtaining statistically significance $(\Delta \mathrm{A}-1.50 \Delta \mathrm{B}-2.10 \mathrm{p}=0.02$ $95 \% \mathrm{CI})$. The RCS was statistically significantly different $(\Delta \mathrm{A}-1.60 \Delta \mathrm{B}-2.50 \mathrm{p}=0,0495 \% \mathrm{CI})$, as was the pain VAS( $\Delta \mathrm{A}-20.80$ $\Delta \mathrm{B}-30.60 \mathrm{p}=0.0495 \% \mathrm{CI}$ ) at week 48. Conclusion: The use of Aminaftone was well tolerated and improved RP as measured by RCS and pain when added to standard of care. A well-designed randomized controlled trial is needed to determine if these preliminary findings are supported.
\end{abstract}

Keywords: Raynaud's Phenomenon, Systemic Sclerosis, Aminaftone, Endotheline

\section{Introduction}

Systemic Sclerosis (SSc) is a chronic autoimmune inflammatory pathogenic disease of the connective tissue, characterized by progressive fibrosis thickening of skin and internal organs. Widespread vasculopathy is one of the hallmarks of SSc and the first vascular event is Raynaud's phenomenon (RP). This is a paroxisymal vasospastic event triggered by cold or emotional states and characterised, in its classic triphasic expression, by pallor (ischaemic phase), followed by cyanosis (cyanotic phase) and lastly redness (reactive hyperaemia phase). The hands are the most frequently affected site, but feet, ears, nose and tongue may also be involved [1]. In the majority of cases, this is a primitive phenomenon, not associated with any disease. In a small percentage, it precedes and/or accompanies disease of the connective tissue, particularly, SSc. In these cases it is defined as secondary Raynaud's phenomenon [2].
While primary RP is characterised by a vasospastic condition that lead to a reduced reactivity to thermal stimuli and never evolves into trophic damage, the RP secondary to $\mathrm{SSc}$ is characterised by an initial vasospastic phase which evolves into intraluminal tissue proliferation, determining a process of tissue ischemia of the arterioles involved [3]. Moreover, RP in SSc patients, often influences the onset of trophic alterations to the fingertips in the form of necrosis, sloughs, ulcers and residual scarring which are subject to infections and, in more severe cases, even amputation; having a deeply negative effect on the quality of life. RP and SSc present a pathophysiological mechanism which is still not completely clear today. According to the so-called "vascular hypothesis," endothelial injury and activation play a central role in the pathogenesis of SSc, and some therapies currently used for SSc are targeted at restoring vascular integrity and homeostasis. Following endothelial damage, local release of cytokines and chemokines activate endothelial cells to upregulate adhesion molecules, such as E-selectin adhesion 
molecule 1 (ELAM-1), intracellular adhesion molecule 1 (ICAM-1), and vascular cell adhesion molecule 1 (VCAM-1), which may eventually shed or be released into the circulation [4,5]. Several studies have highlighted endothelial damage and alterations in the proliferation of vascular structures, with unbalance of factors regulating vascular dilatation and vascular constriction. A very important role appears to be played by endothelin (ET-1), a molecule present in extremely high plasma concentration in these patients and which has a powerful vascular constrictive action [6]. Traditional treatment of RP in SSc consists of the use of calcium channel blockers and prostanoids [7]. Over recent years, the scientific community has focused on some drugs belonging to the endothelin receptor antagonist category and phosphdisterase-5 inhibitors, which have given excellent results for the treatment of pulmonary hypertension and in the treatment of digital ulcers $[8,9,10,11,12]$. Another molecule, Aminaftone, compound

2-hydroxy-3-methyl1-1,4-napthohydroquinone-2-p-aminobe nzoatate $(\mathrm{C} 18-\mathrm{H} 15-\mathrm{N}-\mathrm{O} 4)$ used in the treatment of capillary disorders, has been reported to be effective in reducing vessel permeability and the number and size of leg ulcers in patients with chronic venous insufficiency $[12,13,14]$. Results from preliminary and open label studies suggested that the activity of aminaftone may be related to downregulation of ELAM-1 and VCAM-1 expression and to endothelium remodelling induced by down-regulates ET-1[15,16].

The aim of this study was to assess the efficacy (in terms of improved VAS Pain, RP attacks frequency and Raynaud's Condition Score) of the Aminaftone in patients with Raynaud's Phenomenon secondary to Systemic Sclerosis.

\section{Material and Methods}

In our preliminary study, we evaluated the efficacy of Aminaftone in the treatment of secondary RP in 108 patients with SSc according to the American College of Rheumatology Criteria [17] at least two attacks of Raynaud's Phenomenon, consecutively and divided in two groups matched according to baseline characteristics (age, sex, duration of RP, SSc duration, antibodies, digital ulcers and therapies):

Group A: 51 Patients in treatment with Calcium Channel Blockers, prostanoids (iloprost $2 \mathrm{ng}$ [ $\mathrm{min} / \mathrm{kg}$ every 4 weeks), Endothelin Receptor Antagonist and Phosphodiesterase-5 inhibitors (Standard of Care).

Group B: 57 Patients in treatment with Calcium Channel Blockers, prostanoids, Endothelin Receptor Antagonist and Phosphodiesterase-5 inhibitors and Aminaftone (Standard of care + Aminaftone). Any kind of concomitant medication was allowed, as long as the dose was stable for $\geq 3$ months; no patients receiving moderate or high dose corticosteroids (prednisone $>7,5 \mathrm{mg} /$ day or equivalent) and/or flavonoids were excluded from the study.

Patients with glucose-6-phosphate-dehydrogenase deficiency or other clinical conditions contraindicating the use of Aminaftone were excluded from the study.

Patients were categorized as having limited or diffuse cutaneous SSc according to LeRoy et al. [18] Disease onset was determined by patients' recall of the first non-Raynaud symptom clearly attributable to scleroderma [19].

The use of Aminaftone in this type of patients is according to the indication for which it was registered, the state of capillary fragility. All patients were given a detailed explanation of the study and their informed consent was obtained.

Recruitment of the prospective study was carried out September and October 2013, that means before colder months, in order to place more attention on Raynaud's phenomenon and monitoring it more objectively, during the coldest period of the year.

The analysis and follow up was performed from November 2013 to November 2014

The patients of B group took one tablet of Aminaftone 75 mg twice a day. Visits were planned every 30 days $( \pm 7)$ for 12 months and each patient was given a diary to report on every visit, which indicate:

- Date of onset of Raynaud's Phenomenon.

- Duration: minutes.

- Raynaud's Condition Score (RCS): Limitation of daily activity on a scale of 1 to 10 (meaning 10 as a total inability to do the activity).

- Pain VAS (1-100): 1 meaning the least pain and 100 as the maximum pain number of daily attacks.

- Tingling of fingers (1-100): 1 meaning the least tingling and 100 as the maximum tingling.

- Numbness of fingers (1-100): 1 meaning the least numbness and 100 as the maximum numbness.

- Ulcer onset (date).

- Location ulcer/s.

At each visit, a blood sample was taken for routine analysis in clinical practice and a pregnancy test in women with childbearing potential was performed. Blood count, liver aminotransferase values and creatinine, were also evaluated each month to check the tolerance to the drugs. At the same time, several parameters for disease activity were evaluated: at weeks $0-24^{\text {th }}$ and $48^{\text {th }}$ all patients undergone tests such as Modified Rodnan Skin Score (MRSS) and HAQ disability index (HAQ-DI) [20].

All selected patients were referred to our centre and therefore they already had the diary for at least one year (from which it was possible to extract the information for baseline data)

\section{Statistical Analysis}

The statistical analysis was performed using SPSS statistical software (for windows, version 16.0) and MINI-TAB (for windows, version 14.0). The data were expressed by mean showing the $95 \%$ Cls. $\mathrm{P}<0.05$ was considered statistically significant. Differences in continuous variables between groups were compared via the $U$ Mann-Whitney test for equality. Contrasts between treatment groups with regard to Raynaud symptoms (pain, tingling, numbness) were made using the Wilcoxon Rank-Sum test. 


\section{Results}

106 of 108 patients enrolled completed the study. Two of them were lost to follow up. No significant statistical differences were observed between the 2 groups with respect to demographic and clinical characteristics.

All patients of Group A were aged between 40 and 68 years mean (52.1 \pm SD 10.50). All patients of Group B were aged between 41 and 68 years (mean 51.6 \pm SD 10.20), with SSc according to ACR criteria, presenting RP and DUs with an onset between 6 and 9 months.

Group A: 27 of the 51 patients had the limited form of SSc and 24 of them had a diffuse form. The mid duration of SSc was 8.4 years with 12.8 years of average duration of onset of RP.

Group B: 28 of the 57 patients had the limited form of SSc and 29 of them had a diffuse form. The mid duration of SSc was 7.2 years with 11.2 years of average duration of onset of RP [Tab.1].

In Group A, 6 of the 51 patients completed the diary less than $80 \%$, while in the Group B, 5 of the 57 patients completed the diary less than $80 \%$.

There was no significant difference between the symptomatic manifestations of the two groups of patients at baseline [Tab.2].

Table 1. Details of the demographic and clinical characteristics of patients (tot: 108 pts).

\begin{tabular}{llll}
\hline Sample & Group A & Group B & p-value \\
\hline Patients $\left(\mathrm{n}^{\circ}\right.$, subset D=Diffuse; L=Limited) & $51(24 \mathrm{D} / 27 \mathrm{~L})$ & $57(29 \mathrm{D} / 28 \mathrm{~L})$ & \\
Age, mean \pm SD years & $52.1 \pm \mathrm{SD} 10.50$ & $51.6 \pm$ SD 10.20 & 0.24 \\
Duration RP (mean \pm SD years) & $12.8 \pm 4,4$ & $11,2 \pm 5.1$ & 0.15 \\
SSc Duration since diagnosis (mean \pm SD years) & $8.40 \pm 3.6$ & $7.20 \pm 4.2$ & 0.33 \\
Positive for Scl-70 ab & 25 & 28 & 0.62 \\
Positive for Anticentromere ab & 26 & 29 & 0,54 \\
\hline
\end{tabular}

SD: Standard Deviation; RP: Raynaud's Phenomenon; SSc: Systemic Sclerosis; ab: antibodies

Table 2. Mean Changes from the baseline values in Group A and Group B.

\begin{tabular}{|c|c|c|c|c|c|c|c|c|}
\hline Outcome measures & $\begin{array}{l}\text { Gr. A } \\
\text { baseline }\end{array}$ & $\begin{array}{l}\text { Gr. B } \\
\text { baseline }\end{array}$ & $\begin{array}{l}\text { Gr. A/B p-value } \\
\text { baseline }\end{array}$ & Gr. A 48th wk & Gr. B 48th wk & $\begin{array}{l}\text { Gr. A } \\
\Delta 0-48 \text { th wk }\end{array}$ & $\begin{array}{l}\text { Gr. B } \\
\Delta \text { 0-48th wk }\end{array}$ & $\begin{array}{l}\text { Gr. A/B p-value } \\
\text { 48th wk }\end{array}$ \\
\hline $\begin{array}{l}\text { Average attacks RP } \\
(\mathrm{n} \% \text { day) }\end{array}$ & $3.40 \pm 1.48$ & $3.20 \pm 1.51$ & 0.27 & $1.90 \pm 1.20$ & $1.10 \pm 1.11$ & $(\Delta-1.50)$ & $(\Delta-2.10)$ & 0.02 \\
\hline Duration Attacks (min) & $43.41 \pm 18.78$ & $45.32 \pm 19.29$ & 0.92 & $37,01 \pm 11,56$ & $33,83 \pm 9,71$ & $(\Delta-6.40)$ & $(\Delta-11.50)$ & 0.06 \\
\hline RCS (1-10) & $3.50 \pm 1.41$ & $3.70 \pm 1.32$ & 0.32 & $1.90 \pm 1.31$ & $1.20 \pm 1.10$ & $(\Delta-1.60)$ & $(\Delta-2.50)$ & 0.04 \\
\hline VAS (1-100) & $51.60 \pm 10.05$ & $51.25 \pm 9.38$ & 0.88 & $30,80 \pm 8.93$ & $20,65 \pm 8,75$ & $(\Delta-20.80)$ & $(\Delta-30.60)$ & 0.03 \\
\hline Tingling (1-100) & $33.72 \pm 16.21$ & $32.43 \pm 14.33$ & 0.95 & $22.14 \pm 11.61$ & $13.48 \pm 8.22$ & $(\Delta-11.58)$ & $(\Delta-18.95)$ & 0.04 \\
\hline Numbness (1-100) & $41.36 \pm 24.10$ & $42.22 \pm 28.16$ & 0.81 & $30.76 \pm 19.40$ & $25.34 \pm 18.82$ & $(\Delta-10.60)$ & $(\Delta-16.88)$ & 0.05 \\
\hline MRSS & $17.10 \pm 8.10$ & $15.90 \pm 9,30$ & 0.89 & $15.80 \pm 7.20$ & $14.70 \pm 8.30$ & $(\Delta-1.30)$ & $(\Delta-1.20)$ & 0.30 \\
\hline HAQ-DI & $1.13 \pm 0.21$ & $1.07 \pm 0.20$ & 0.37 & $0.95 \pm 0.19$ & $0,83 \pm 0.19$ & $(\Delta-0.18)$ & $(\Delta-0.24)$ & 0.09 \\
\hline DUs & $0.54 \pm 0.66$ & $0.51 \pm 0.66$ & 0.87 & $0,32 \pm 0.48$ & $0.22 \pm 0.44$ & $(\Delta-0.22)$ & $(\Delta-0.29)$ & 0.08 \\
\hline
\end{tabular}

RP: Raynaud's Phenomenon; RCS Raynaud's Condition Score; MRSS: Modified Rodnan Skin Score; HAQ-DI: Scleroderma Health Assessment Questionnaire-Disability Index; DUs: Digital Ulcers

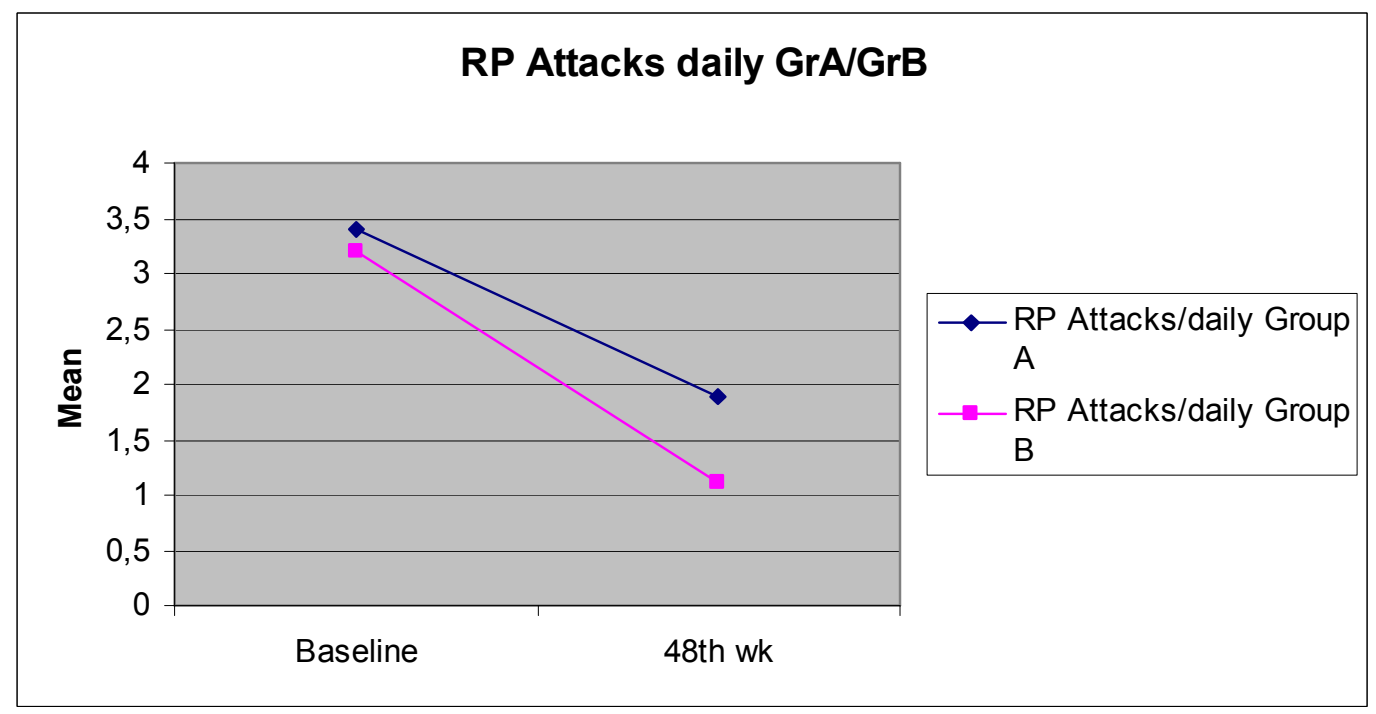

Fig. 1. The mean changes from the baseline of Raynaud's Phenomenon attack frequency. 


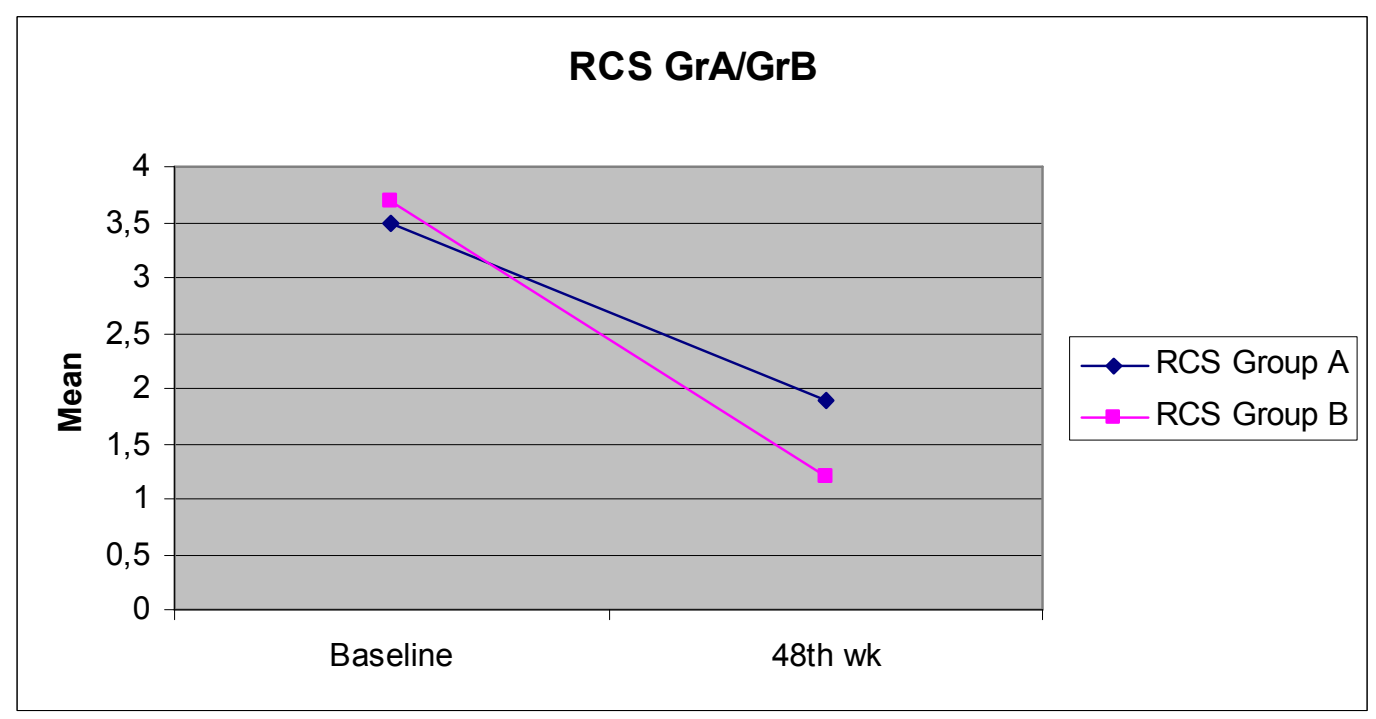

Fig. 2. The mean changes from the baseline of Raynaud's Condition Score.

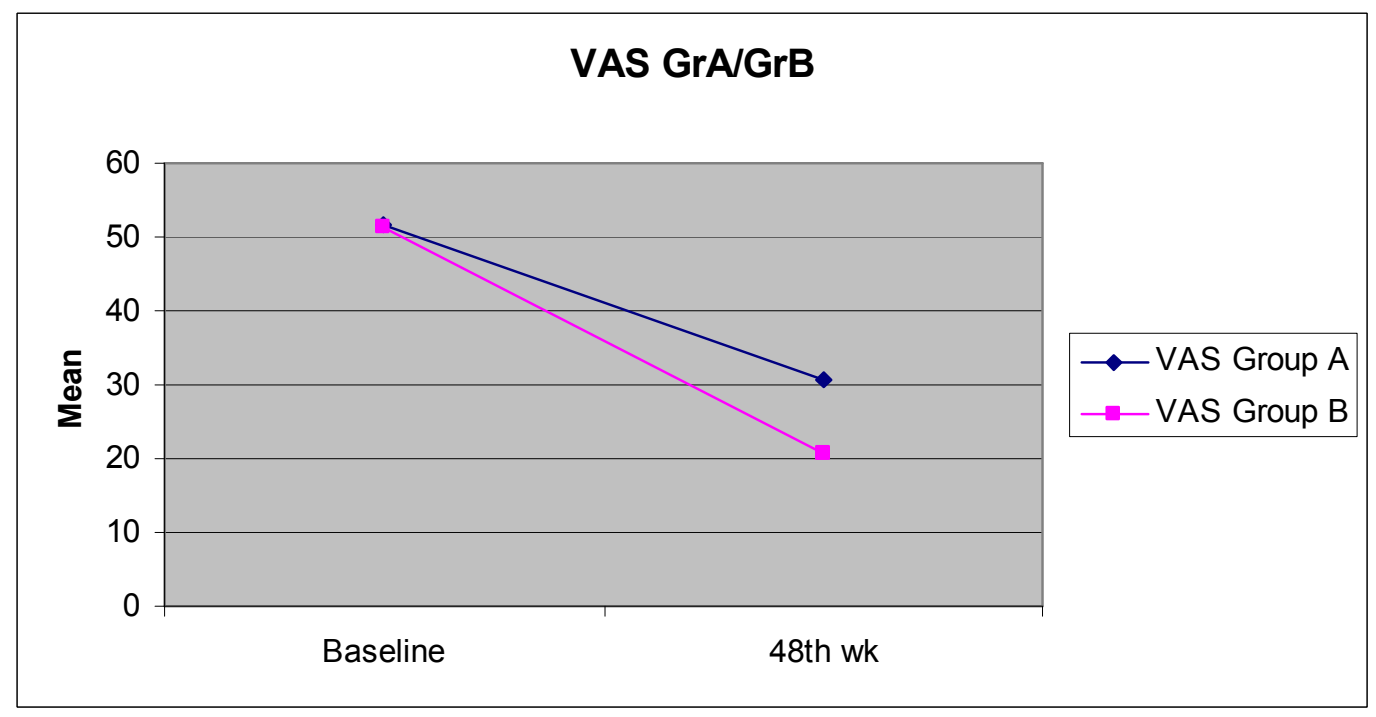

Fig. 3. The mean changes from the baseline of Visual Analogic Scale Pain.

The number of RP attacks in group treated with Aminaftone (Group B) was lower than group treated with standard therapy (Group A) from baseline to Week 48, obtaining statistically significance $(\Delta \mathrm{A}-1.50 \Delta \mathrm{B}-2.10 \mathrm{p}=0.0295 \% \mathrm{CI})$ [Fig.1].

The duration of the RP was decreased, but did not obtain statistically significance $(\Delta \mathrm{A}-6.40 \Delta \mathrm{B}-11.50 \mathrm{p}=0,0995 \%$ $\mathrm{CI})$. The RCS has shown a statistically significant improvement $(\triangle \mathrm{A}-1.60 \Delta \mathrm{B}-2.50 \mathrm{p}=0.0495 \% \mathrm{CI})$ [Fig.2], as well as the pain VAS $(\Delta \mathrm{A}-20.80 \Delta \mathrm{B}-30.60 \mathrm{p}=0.0495 \% \mathrm{CI})$ at week 48 [Fig.3]. The Tingling and Numbness scale have shown a statistically significant improvement $(\Delta \mathrm{A}-11.58 \Delta \mathrm{B}$ $-18.95 \mathrm{p}=0.0495 \% \mathrm{CI} ; \Delta \mathrm{A}-10.60 \Delta \mathrm{B}-16.88 \mathrm{p}=0.0595 \% \mathrm{CI})$ The number of DUs decreased in both group of patients $(\triangle \mathrm{A}$ $-0.22 \Delta \mathrm{B}-0.29 \mathrm{p}=0.0995 \% \mathrm{CI})$. Furthermore, in patients treated with Aminaftone the number of the onset of new DUs was lower than to the untreated group. Specifically even in patients with ERA and aminaftone the number of new DUs was lower than to patients treated with only ERA.
There were no differences in the MRSS $(p=0.30)$ and HAQ-DI ( $p=0.09)$ [Tab.2]. There were not serious adverse events during the study, only in two patients there was an increase of liver transaminases (2-fold upper limit of normal), one patient had episodes of low blood pressure and another patient presented peripheral edema, but none of these were in Group B (standard care + Aminaftone).

\section{Discussion}

This is the first open label controlled cohort study with patients treated with Aminaftone in association to standard therapy for secondary Raynaud's Phenomenon in SSc. Our data showed a statistically significant improvement of baseline in the number of attacks of RP, VAS pain, Tingling, Numbness and RCS. Same results have been highlighted by other studies where endothelin receptor antagonists were used $[21,22,23]$. Few reports described the successful of 
Aminaftone but these are studies on a small sample of patients [20], while there are some studies (but non-blinded approach) of bosentan in the treatment of Raynaud's Phenomenon and digital ulcers in SSc patients $[15,25,26,27]$. Thus, this preliminary study it's restricted by its limitations. It is too underpowered to detect small treatment effect. Nevertheless as previously shown by other authors in treatment with ERA and PHE-5 Inhibitors, our data highlighted a statistically significant improvement of RP $[12,21,27,28]$, while another studies failed to document any beneficial effect on VAS, RP or development of digital ulcers [29,30,31]. These phenomena determine patient worsening of the quality of life, not only in terms of pain, but also due to the extreme difficulty in performing normal daily activities. A very interesting phenomenon was that some patients with oral mucositis (6 women) have improved until complete resolution of symptoms during treatment with Aminaftone. This is probably related to the reduction of the edematous phase that this molecule has been shown to has in several studies [13]. Our results have highlighted the possible effectiveness of Aminaftone in association with standard therapy resulting in a synergic effect on vasospastic phenomena, which confirm the fundamental role of endothelin in the pathogenesis of SSc and her different level of pathway, even if the data remain still unclear. Moreover Aminaftone has an effect of down-regulation of many cytokines including for example IL-6, which intervenes in the acute inflammatory phase. Also decreases levels of TGF- $\beta$ which can lead to pulmonary fibrosis activating fibroblasts to produce excessive collagen deposition.

This study has some limitations. It's not randomized, not blind and the method of data collection is not accurate (diary completed by the patient).

\section{Conclusion}

Aminaftone was well tolerated and improved RP as measured by RCS and pain when added to standard of care. A well-designed randomized controlled trial is needed to determine if these preliminary findings are supported.

\section{References}

[1] Kahaleh MB. Raynaud phenomenon and the vascular disease in scleroderma. Curr Opin Rheumatol 2004;16: 718-22.

[2] Wigley FM. Clinical practice. Raynaud's Phenomenon. N Engl J Med 2002;347:1001-8

[3] Herrick AL. Pathogenesis of Raynaud's phenomenon. Rheumatology 2005;44:587-96.

[4] Blann A et al. Soluble markers of endothelial cell function. Clin Hemorhed Microcirc. 1997;17:3-11.

[5] Rothlein R et al. A form of circulating ICAM-1 in human serum. J Immunol. 1991;147:3788-3793.

[6] Mayes MD. Endothelin and endothelin receptor antagonists in systemic rheumatic disease. Arthritis Rheum 2003; 48:1190-9.

[7] Black CM, Denton CP. The management of systemic sclerosis. Br J Rheumatol 1995;34:3 7.

[8] Della Rossa A et al. Oral sildenafil in skin ulcers secondary to systemic sclerosis. Scand J Rheumatol. 2011;40(4):323-5. doi: 10.3109/03009742.2011.569755. Epub 2011 Jun 6. No abstract available.

[9] Korn JH, Mayes M, Matucci Cerinic M et al. Digital ulcers in systemic sclerosis: prevention by treatment with bosentan, an oral endothelin receptor antagonist. Arthritis Rheum 2004;50:3985-93.

[10] Matucci-Cerinic M, Denton CP, Furst DE et al. Bosentan reduces the number of new digital ulcers in patients with systemic sclerosis (abstract). Ann Rheum Dis 2006;65: A3-49.

[11] Parisi S et al. Efficacy of ambrisentan in the treatment of digital ulcers in patients with systemic sclerosis: a preliminary study. Rheumatology (Oxford). 2013 Jun;52(6):1142-4. doi: 10.1093/rheumatology/ket019. Epub 2013 Mar 5.

[12] Villaverde CA et al. About vascular permeability with amonaftone. Revista de farmacologia Clinica y Experimental. 1989;6:9-14.

[13] De Anna D et al. Effects of therapy with aminaftone on chronic venous and lymphatic stasis. Minerva Cardioangol. 1989;37:251-254.

[14] Pereira de Godoy JM. Treatment of stasis dermatitis using aminaphtone: a case series. Journal of Medical Reports 2010,4:295.

[15] Zambelli V et al. Efficacy of aminaftone in a rat model of monocrotaline-induced pulmonary hypertension. Eur J Pharmacol. 2011 Sep 30;667(1-3):287-91. doi: 10.1016/j.ejphar.2011.05.060. Epub 2011 May 30.

[16] Scorza R et al. Aminaftone, a derivative of 4-aminobenzoic acid, downregulates endothelin-1 production in ECV304 Cells: an in vitro Study. Drugs R D. 2008;9(4):251-7.

[17] Subcommittee for Scleroderma Criteria of the American Rheumatism Association Diagnostic and Therapeutic Criteria Committee. Preliminary criteria for the classification of systemic sclerosis (scleroderma). Arthritis Rheum 1980;23:581-90.

[18] LeRoy EC et al. Scleroderma (Systemic Sclerosis): Classification, subsets and pathogenesis. J Rheumatol. 1988;15:202-205.

[19] White et al. The American College of Rheumatolgy Committee on Design and Outcomes in Clinical Trials in Systemic Sclerosis. Guidelines for Clinical Trials in Systemic Sclerosis. I. Disease-modifying interventions. Arthritis Rheum. 1995;38:351-360.

[20] Merkel PA, Herlyn K, Martin RW et al. Scleroderma Clinical Trials Consortium. Measuting disease activity and functional status patients with scleroderma and Raynaud's phenomenon. Arthritis Rheum 2002;46:2410-20.

[21] Garcia de la Pena-Lefebvre P, Rodri'guez Rubio S, Valero Exposito $\mathrm{M}$ et al. Long-term experience of bosentan for treating ulcers and healed ulcers in systemic sclerosis patients. Rheumatology 2008;47:464-6. 
[22] Selenko-Gebauer N, Duschek N, Minimair G, Stingl G, Karlhofer F. Successful treatment of patients with severe secondary Raynaud's phenomenon with the endothelin receptor antagonist bosentan. Rheumatology 2006; 45(Suppl. 3):iii45-8.

[23] Kamata Y, Minota S. Effects of phosphodiesterase type 5 inhibitors on Raynaud's phenomenon. Rheumatol Int. 2014 May 1. [Epub ahead of print].

[24] Scorza R et al. Effects of aminaftone $75 \mathrm{mg}$ TID on soluble adhesion molecules: a 12-week, randomized, open-label pilot study in patients with systemic sclerosis. Clin Ther. 2008 May;30(5):924-9. doi: 10.1016/j.clinthera.2008.05.009.

[25] Ramos-Casals M, Brito-Zero' n P, Nardi N et al. Successful treatment of severe Raynaud's phenomenon with bosentan in four patients with systemic sclerosis. Rheumatology 2004;43:1454-6.

[26] Yu EP, Ostor AJ, Hall FC. Successful treatment with bosentan for severe digital ischaemia in limited cutaneous systemic sclerosis. Ann Rheum Dis 2007;66: 1122-3.

[27] Parisi S et al. Efficacy of bosentan in the treatment of
Raynaud's phenomenon in patients with systemic sclerosis never treated with prostanoids. Reumatismo. 2014 Mar 17;65(6):286-91. doi: 10.4081/reumatismo.2013.691.

[28] Launay D, Diot E, Pasquier E et al. [Bosentan for treatment of active digital ulcers in patients with systemic sclerosis]. Presse Med 2006;35:587-92.

[29] Nguyen VA, Eisendle K, Gruber I, Hugl B, Reider D, Reider N. Effect of the dual endothelin receptor antagonist bosentan on Raynaud's phenomenon secondary to systemic sclerosis: a double-blind prospective, randomized, placebo-controlled pilot study. Rheumatology (Oxford). 2010 Mar;49(3):583-7. Epub 2009 Dec 29.

[30] Seibold JR, Matucci-Cerenic M, denton CP, Furst DE, Mayer $\mathrm{MD}$, Kramer $\mathrm{F}$ et al. Bosentan reduces the number of new digital ulcers in patients with systemic sclerosis. Ann Rheum Dis. 2006;65:90.

[31] Moore TL, Vail A, Herrick AL. Assessment of digital vascular structure and function in response to bosentan in patients with systemic sclerosis-related Raynaud's phenomenon. Rheumatology 2007;46:363-4. 